\title{
Estructura de un BOSQUe de RAMORINOA GIROLAE (FABACEAE), EN la Sierra de Pie de Palo, San Juan (Argentina)
}

\author{
MARTÍN HADAD ${ }^{1,2}$, MARTÍN ALMIRON ${ }^{1}$ y JUAN SCAGLIA ${ }^{3}$
}

\begin{abstract}
Resumen: Ramorinoa girolae Speg. (Fabaceae), es una especie leñosa endémica de Argentina comúnmente llamada "Chica", considerada vulnerable por su restringida distribución geográfica, lento crecimiento y escasa resistencia al fuego. En el presente trabajo se estudió la estructura y el estado sanitario de una población de $R$. girolae en San Juan, Argentina. Se muestrearon 94 individuos en 8 parcelas de $1000 \mathrm{~m}^{2}$ cada una (densidad promedio por parcela: 117,5 árboles ha-1) y se clasificó el estado sanitario de los árboles en tres categorías: "muy bueno", "bueno" y "malo". La condición general del bosque fué buena con $31,91 \% ; 57,44 \%$ y $9,57 \%$ del total de los ejemplares en cada categoría. El mayor número de árboles se registró en la posición topográfica de ladera $(N=55)$. La altura promedio de los árboles fue de $4,05 \pm 1,47 \mathrm{~m}$ con un alto número de árboles semi-decumbentes $(N=39)$. El mayor número de árboles se registró entre los $10-40 \mathrm{~cm}$ de diámetro basal y sólo tres renovales con un diámetro basal inferior a $10 \mathrm{~cm}$, lo que sugiere que el bosque no habría regenerado en las últimas décadas. Para futuros planes de manejo y conservación, se sugiere investigar los patrones de regeneración $R$. girolae, con el objetivo de identificar factores ecológicos clave para la conservación in situ de esta especie.
\end{abstract}

Palabras clave: Diámetro basal, conservación, Ramorinoa girolae, especie vulnerable, estructura de bosque.

Summary: Ramorinoa girolae (Fabaceae) forest structure in Sierra de Pie de Palo, San Juan (Argentina). Ramorinoa girolae Speg. (Fabaceae), commonly called "Chica", is a small tree endemic to Argentina. Owing to its restricted geographical distribution, slow growth, and poor fire resistance, $R$. girolae was categorized as vulnerable. In the present study, the size structure and health status of a $R$. girolae population was analyzed in the Pie de Palo hill, San Juan, Argentina. We sampled 94 trees in eight 1000 $\mathrm{m}^{2}$ plots (average plot density 117.5 trees $\mathrm{ha}^{-1}$ ), and classified the health status of each tree into three categories: "very good", "good" and "bad". In general, the health condition of R. girolae was good, with $31.91 \%, 57.44 \%$ and $9.57 \%$ of the trees in each category, respectively. The highest number of trees was found in the topographic 'slope' position $(N=55)$. Average tree height was $4.05 \pm 1.47 \mathrm{~m}$ with a high number of semi-decumbent trees $(N=39)$. The highest number of individuals was recorded between 10-40 $\mathrm{cm}$ basal diameter and only three offshoots were less than $10 \mathrm{~cm}$. This observation suggests an overall lack of "chica" regeneration in the last decades. Future conservation and management plans should aim to investigate regeneration patterns of $R$. girolae in order to identify key ecological factors for in situ conservation of this species.

Key words: Basal diameter, conservation, Ramorinoa girolae, vulnerable species, woodland structure.

\footnotetext{
1 Departamento de Biología (FCEFyN), Universidad Nacional de San Juan, Consejo Nacional de Investigaciones Científicas y Tecnológicas (CONICET).

2 Departamento de Dendrocronología e Historia Ambiental. IANIGLA, CCT-CONICET, Mendoza.

${ }^{3}$ Banco de Germoplasma. Instituto de Investigación y Desarrollo Agroindustrial Hortícola Semillero (San Juan). mhadad@mendoza-conicet.gov.ar; martinalmiron2000@hotmail.com; joposcaglia@gmail.com
} 


\section{INTRODUCCIÓN}

A escala de paisaje, la estructura de un bosque es el resultado de las interacciones bióticas y abióticas (Romme \& Knight, 1982; Archer, 1994). La dinámica del bosque, tal como puede ser interpretada por la estructura de la población y patrón de reclutamiento, se encuentra influida por muchos procesos, incluyendo perturbaciones ambientales e interacciones entre los árboles (North et al., 2004; Taylor et al., 1996). La interpretación de la distribución de tamaños o edades en un rodal, da indicios acerca de cómo ha sido el establecimiento de los árboles en el pasado (Veblen, 1992; Duncan, 1993; Cullen et al., 2001), por lo que la estructura de diámetros y/o edad de un bosque es el reflejo de la historia de vida de los árboles, resultante de la combinación de natalidad y mortalidad en el tiempo (Brown \& Wu, 2005). En este sentido, el estudio de la estructura de edades o diamétricas, ayudarían a comprender los procesos que determinan la estructura de la población a través del tiempo (Svensson \& Jeglum, 2001). Las mediciones de diámetro del tallo son ampliamente utilizadas en ecología y gestión forestal para caracterizar la distribución del tamaño de las masas forestales y para estimar los volúmenes de madera (Newton, 2007). Existen numerosos trabajos que utilizan esta metodología para conocer la estructura y dinámica del bosque (e.j. Dezzotti \& Sancholuz; 1991; Villegas et al., 2003; Bannister et al., 2008). Para el Monte Argentino se pueden mencionar al respecto los trabajos de Alvarez et al. (2006), Gil (2013) y Martínez de Escobar et al. (2013). Las reconstrucciones cuantitativas de la estructura del bosque (distribución y rango de edades y/o diámetro de los árboles), también podrían ser un punto de referencia para la restauración y gestión de los ecosistemas forestales. Por lo tanto, evaluar y analizar la estructura del bosque es uno de los requisitos previos para la comprensión de los procesos ecológicos y de restauración de los bosques naturales (Wang et al., 2004).

En la provincia de San Juan se dispone de 2.248.416 ha bajo distintas categorías de áreas naturales protegidas (Marquez, 1999; Dirección de Conservación y Áreas Protegidas de San Juan, 2009). Una de estas áreas es la Reserva de Usos Múltiples Valle Fértil (Ley Prov. $\mathrm{N}^{\circ}$ $3.666 / 71$ ) que cuenta con 800.000 ha. Esta reserva es de gran interés biogeográfico por su carácter ecotonal, mostrando la transición entre la provincia Fitogeográfica del Monte con la del Chaco Serrano. Una parte importante de la superficie de dicha área protegida incluye el macizo denominado "Sierra de Pie de Palo" (3000 msm) ubicado al sudoeste del área protegida. Esta formación representa una isla biogeográfica, con especies vegetales representativas de diversos ambientes (Kiesling, 1994, 2003 y 2009; Dirección de Conservación y Áreas Protegidas de San Juan, 2009), en el cual se pueden encontrar bosques de Ramorinoa girolae en algunas quebradas de dicha formación.

Ramorinoa girolae Speg. (Fabaceae), es una especie endémica de Argentina comúnmente llamada "Chica", única integrante del género Ramorinoa. Esta especie forma bosquecillos cuya distribución es fragmentada y restringida a los faldeos montañosos de San Luís, San Juan y La Rioja, en el cuadrante delimitado por los paralelos $29^{\circ} 30^{\prime}$ y $33^{\circ} 00^{\prime} \mathrm{S}$, y los meridianos $57^{\circ} 00^{\prime}$ y $58^{\circ}$ 00' W (Hunziker \& Coccucci, 1961; Beorchia Nigris, 2001; Demaio et al., 2002). En cuanto a su poder germinativo, Meglioli et al. (2012) mostraron que el escarificado químico con ácido sulfúrico puro resultó ser un tratamiento efectivo para poder romper con la dormición. Por otro lado, Demaio et al. (2002) mencionan que los pobladores consumen la semilla crudas o tostadas y que también son consumidas por roedores silvestres, además, Zapata et al. (2010) informan que las semillas tienen un importante valor nutricional, conteniendo un alto porcentaje de proteínas $(21,2 \%)$ y lípidos (29,3\%). En un trabajo reciente Luna et al. (2013) reportan que el contenido fenólico y la actividad antioxidante de las semillas son un indicador de su capacidad para ofrecer beneficios potenciales para la salud. Por otro lado, la madera de $R$. girolae es una de las más duras, con una densidad de 1,265 $\mathrm{kgdm}^{-3}$ (Kiesling, 1994). Las razones por las que se consideran a $R$. girolae una especie "Vulnerable" son su restringida distribución geográfica, lento crecimiento y poca resistencia al fuego, entre otras (Demaio et al., 2002). Actualmente se encuentra en proceso de validación para incluirla en la categoría "En peligro" (EN) por cumplir con los criterios $\mathrm{B}_{2} \mathrm{ab}$ (iii) del protocolo de la International Union for Conservation of Nature (IUCN) (Demaio, com pers).

Esta especie ha sido poco estudiada desde el 
punto de vista ecológico en general y forestal particularmente. Sumado a esta falta de información, no existen antecedentes sobre el estado actual de los bosques de $R$. girolae, por lo que resulta importante conocer y conservar los bosques fragmentados de esta especie. El objetivo del presente trabajo es analizar la estructura de un bosque de $R$. girolae en la Sierra de Pie de Palo, San Juan, teniendo en cuenta la topografía en donde se encuentra. Esta información nos permitirá conocer más sobre su ecología y su ambiente donde se desarrolla y poder generar posibles pautas de manejo.

\section{Materiales y Método}

\section{Área de estudio}

El área de estudio se encuentra inmersa en el sector sur de la Sierra de Pie de Palo en la localidad de Vallecitos, del departamento Caucete $\left(31^{\circ} 42^{\prime}\right.$ $\left.15,5^{\prime \prime} \mathrm{S} ; 68^{\circ} 00^{\prime} 47,8^{\prime \prime} \mathrm{W}\right)$, a $60 \mathrm{~km}$ al este de la Capital de San Juan (Fig. 1); altitudinalmente el área de estudio se encuentra entre los 790 a 929 m s.n.m. Esta área se ubica dentro de la Provincia Fitogeográfica del Monte (Cabrera, 1994), climáticamente dominado por condiciones áridas y semi-áridas (Labraga \& Villalba, 2009), con precipitaciones medias anuales entre los 30 a $350 \mathrm{~mm}$ y una temperatura media anual entre los $13^{\circ}$ y $15,5^{\circ}$ (Morello, 1958). La vegetación presente en el área de estudio posee componentes en tres estratos, en los que el arbóreo está representado principalmente por $R$. girolae, Prosopis flexuosa var. flexuosa D.C y Maytenus viscifolia Griseb. El estrato arbustivo corresponde a un jarillal de Larrea divaricata Cav., Zuccagnia punctata Cav., Aloysia sp. y Monttea aphylla (Miers) Benth. \& Hook., entre otras. En el estrato herbáceo, se observan gramíneas como Pappophorum caespitsum R.E. Fr., P. philippianum Parodi, Trichloris crinita (Lag.) Parodi y Aristida mendocina Phil.

\section{Descripción botánica de Ramoriona girolae}

Ramorinoa girolae es un arbusto o árbol pequeño que puede crecer hasta los $10 \mathrm{~m}$ de altura, posee tronco generalmente entre $10-20 \mathrm{~cm}$ de diámetro. Posee una copa globosa, es completamente áfila y presenta características xerófilas. Las ramas son cilíndricas erguidas, rectas, de color verde pálido y terminan en espina. Sus flores son amarilloanaranjadas de $1 \mathrm{~cm}$ de longitud. El fruto es una legumbre leñosa, de color castaño oscura, de 3-6

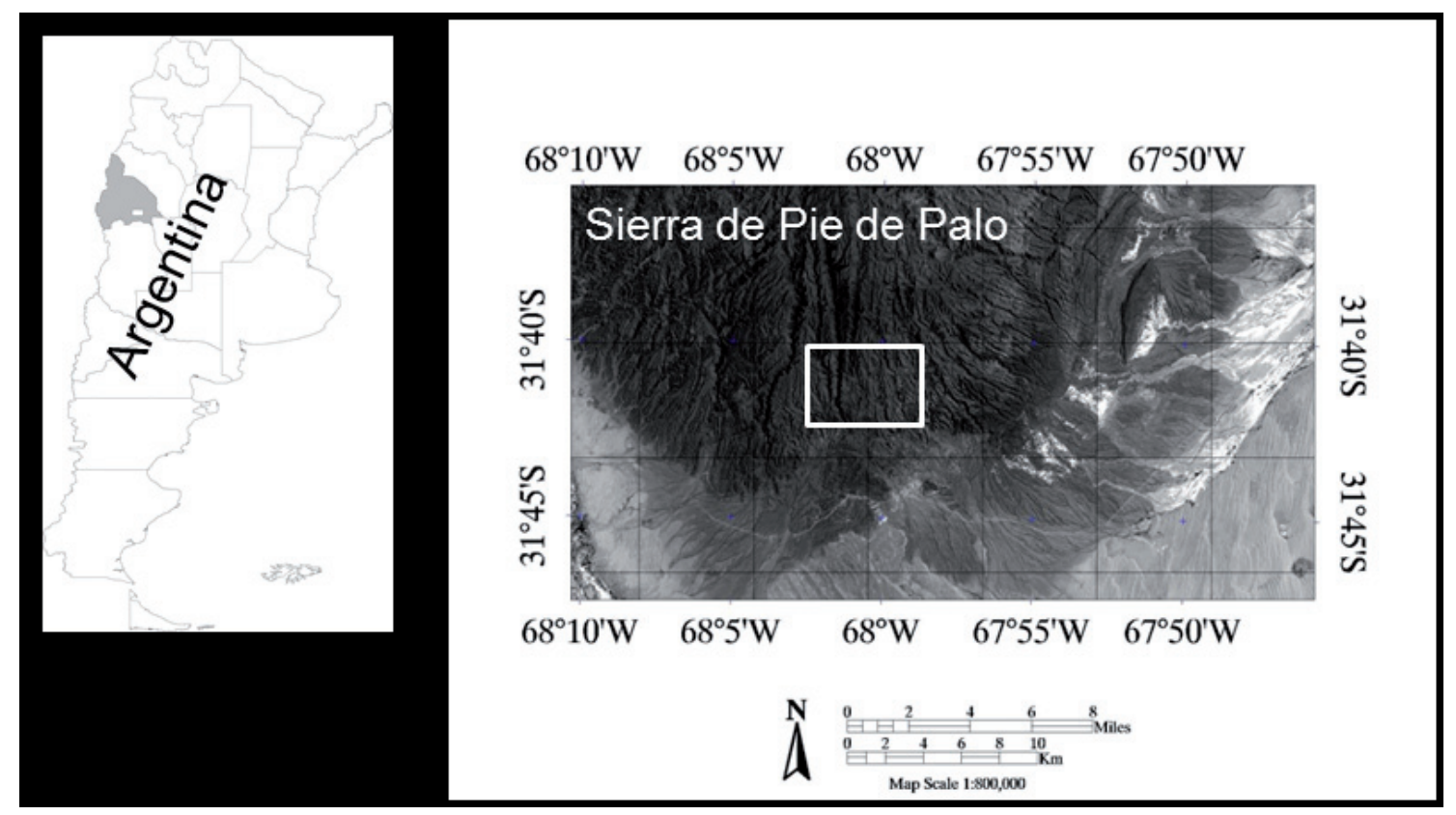

Fig. 1: El cuadro blanco representa la ubicación geográfica del bosque Ramorinoa girolae en la Sierra de Pie de Palo, en la provincia de San Juan, Argentina. 
$\mathrm{cm}$ de longitud. En cuanto a su fenología florece a partir de septiembre hasta noviembre y los frutos maduran entre febrero y marzo (Kiesling, 1994, Cangiano et al., 1998, Demaio et al., 2002).

\section{Diseño de muestreo}

La topografía del área está constituida principalmente por numerosas quebradas de dirección norte-sur, con un sistema hidrológico de carácter temporario. Para asegurar la independencia entre los relevamientos, se seleccionaron 8 quebradas separadas entre sí por más de $100 \mathrm{~m}$. En cada quebrada se implementó una parcela de 1.000 $\mathrm{m}^{2}(50 \times 20 \mathrm{~m})$ que atravesaba toda la quebrada, desde su cima, hasta la base. Dentro de cada parcela, se observaron tres ambientes clasificados en: cima, a la parte superior de la quebrada; ladera, a la parte media de la quebrada y en cauce, periferia de los cauces secos. Esto permite garantizar la presencia de $R$. girolae en todas la secciones de la quebrada, a fin de caracterizar el ambiente donde es más abundante. Para evaluar si hay un efecto de la posición topográfica en la distribución de los individuos y si las parcelas son homogéneas entre sí, se realizó un análisis de la varianza y separación de media a través del test de Tuckey considerando a la posición topográfica y a la parcela como factores. Para cumplir los supuestos de homocedasticidad y normalidad el número de individuos por ambientes fue transformado mediante la raíz cuadrada.

En cada parcela se registró la totalidad de individuos de $R$. girolae para estimar la densidad de individuos por hectárea. A su vez, en cada árbol se contó el número de fustes y se midió el diámetro basal, con lo que se calculó el basal (DAB), para esto se utilizó la siguiente fórmula (Alvarez et al. 2006):

$\mathrm{DAB}=2 \sqrt{ }\left\{\pi\left(\mathrm{dab}_{1} / 2\right)^{2}+\pi\left(\mathrm{dab}_{2} / 2\right)^{2}+\pi\left(\mathrm{dab}_{3} / 2\right)^{2}+\ldots .+\right.$ $\left.\pi\left(\mathrm{dab}_{\mathrm{n}} / 2\right)^{2}\right\} / \pi$

donde el dab ${ }_{1}$, dab $_{2}, \ldots$, dab $_{\mathrm{n}}$ es el diámetro basal de cada fuste. Con esta información se pudo obtener una frecuencia diamétrica de distintas clases de DAB.

También se midió la altura de los árboles y los diámetros mayor (DMaC) y menor de copa (DMeC). Para obtener la superficie de copa, se empleó la fórmula del óvalo $[\pi(\mathrm{DMaC} / 2)(\mathrm{DMeC} / 2)]$. Además se clasificó la forma de cada árbol en: erectos, semidecumbentes y decumbentes. Los árboles que se consideraron erectos presentaban un crecimiento vertical, los semidecumbentes a aquellos individuos cuyas ramas secundarias presentaron un crecimiento cercano a la horizontal o hacia abajo pero que no llegaran al suelo. Por último se consideraron decumbentes a los individuos cuyas ramas laterales péndulas alcanzaron el suelo (Pasiecznik et al., 2001). Tanto para los valores de altura, DAB y diámetro de copa mayor y menor se calculó su valor medio y desviación estándar (DS).

También se observó el estado sanitario de los árboles. Para esto, se los clasificó en tres categorías según la estimación del porcentaje total de ramas vivas y con buen vigor en: a) Muy Bueno, cuando el árbol presentaba más del $80 \%$ de la copa con ramas vivas y con buen vigor; b) Bueno, cuando el árbol presentaba entre el 40 y el $80 \%$ de la copa con ramas secas; c) Mal Estado, cuando el árbol presentaba menos del 40\% de la copa con ramas secas (Según Álvarez et al., 2006 modificado). Con la finalidad de comparar el DAB con la altura y el diámetro de copa para saber si existía alguna respuesta a esta variable, se realizó un análisis mediante una regresión lineal. Se utilizó al DAB como variable independiente mientras que la altura y el diámetro de copa fueron las variables dependientes.

\section{Resultados}

Se registraron 94 individuos en las 8 parcelas estudiadas $\left(8000 \mathrm{~m}^{2}\right)$ y se determinó una densidad media de 117,5 árboles ha-1 ${ }^{-1}$ La posición topográfica del terreno influyó significativamente en la distribución de los ejemplares observados. El mayor número de árboles se encontró en la ladera, (55), seguido por los ubicados en el cauce (28) y en la cima solamente 11 individuos (Tabla 1). El número de individuos por parcelas resulto homogéneo ya que no se encontró diferencia significativa en el análisis $(F=0,15 ; P=0,99)$. En cuanto al estado de sanidad del bosque se observó que el 31,91\% de los árboles presentaron un estado Muy Bueno y el 57,44\% en estado Bueno y el $9,57 \%$ en estado Malo.

Para la distribución por clases de altura, se registró el mayor número de árboles entre los dos y cuatro metros, siendo la clases de tres metros la que mayor número de individuos presentó (Fig. 2) y la altura promedio de los árboles del bosque

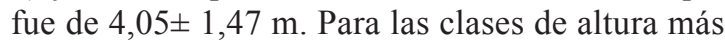


Tabla 1: Análisis de la varianza y separación de medias por test de Tuckey $(P<0,05)$ del

número de individuos de $R$. girolae por posición topográfica (Cima, Cauce y ladera).

\begin{tabular}{|c|c|c|}
\hline $\begin{array}{l}\text { Posición } \\
\text { Topográfica }\end{array}$ & Medias & $(F=3,72 ; P=0,041)$ \\
\hline Cima & 0,72 & $A$ \\
\hline Cauce & 1,55 & $A B$ \\
\hline Ladera & 2,3 & $B$ \\
\hline
\end{tabular}

Valores medios corresponden a las transformaciones mediante la Raíz cuadrada. Letras distintas indican diferencias significativas $(P \leq 0,05)$.

bajas, se observó solamente un individuo de $1 \mathrm{~m}$ $\mathrm{y}$ dos renovales inferiores a $1 \mathrm{~m}$ de altura. Por otro lado, para las clases de altura de $7 \mathrm{~m}$ y $8 \mathrm{~m}$, se contabilizaron sólo dos árboles para cada una de ellas. Se registró mayor número de individuos con forma semidecumbente $(N=39)$, seguido por decumbente $(N=33)$ y erecto $(N=22)$. Cuando se relacionó la forma del árbol con la altura en la clase de $3 \mathrm{~m}$ y $4 \mathrm{~m}$, la mayoría de los individuos presentó forma semidecumbente y decumbente (Fig. 2). Se observó que los árboles erectos no superaron los $4 \mathrm{~m}$ de altura, y que se presentaron en mayor proporción en las categorías menores a los 3m (Fig. 2). La distribución diamétrica de
$R$. girolae se asemeja a una curva exponencial negativa, lo cual indicaría un establecimiento continuo, sin embargo se encontró solamente tres individuos menores de $10 \mathrm{~cm}$ de diámetro basal. El diámetro basal medio fue de 35,89 $\pm 19,15 \mathrm{~cm}$ y se registró que el mayor número de individuos se encontró entre los $10-40 \mathrm{~cm}$ de diámetro basal (Fig. 3). También se midieron tres árboles con ancho de diámetro mayor a $80 \mathrm{~cm}$. Al relacionar las clases de diámetros con la posición topográfica se observó que en las clases comprendidas entre 10 a $60 \mathrm{~cm}$ prevalecen los árboles que se encuentran en la ladera, seguidos de los que se encuentran en el cauce. Las clases diamétricas más grandes presentaron un mayor número de individuos en la cima (Fig. 3A). En cuanto al número de fustes, en casi todas las clases diamétricas se observaron árboles con un solo fuste $(N=53)$, seguidos de árboles con dos fustes $(N=28)$, los demás árboles presentaron tres, cuatro y cinco fustes $(N=10 ; N=2$ y $N=1$ respectivamente) (Fig. $3 \mathrm{~B})$.

La relación entre la altura y el diámetro basal marcó una tendencia lineal positiva $\left(R^{2}=0,31 ; F=\right.$ 41,$81 ; P=0,0001 ;$ Fig. 4). Además presentó una gran dispersión el diámetro basal en los árboles cuyas alturas superaron los $3 \mathrm{~m}$. En este sentido, se puede observar árboles que presentan un $\mathrm{DAB}$ de $0,60 \mathrm{~m}$ y pueden tener entre 4 y 8 metros de altura. En cuanto a los diámetros de copa, el promedio del diámetro mayor fue de $8,33 \pm 4,16 \mathrm{~m}$ y del menor

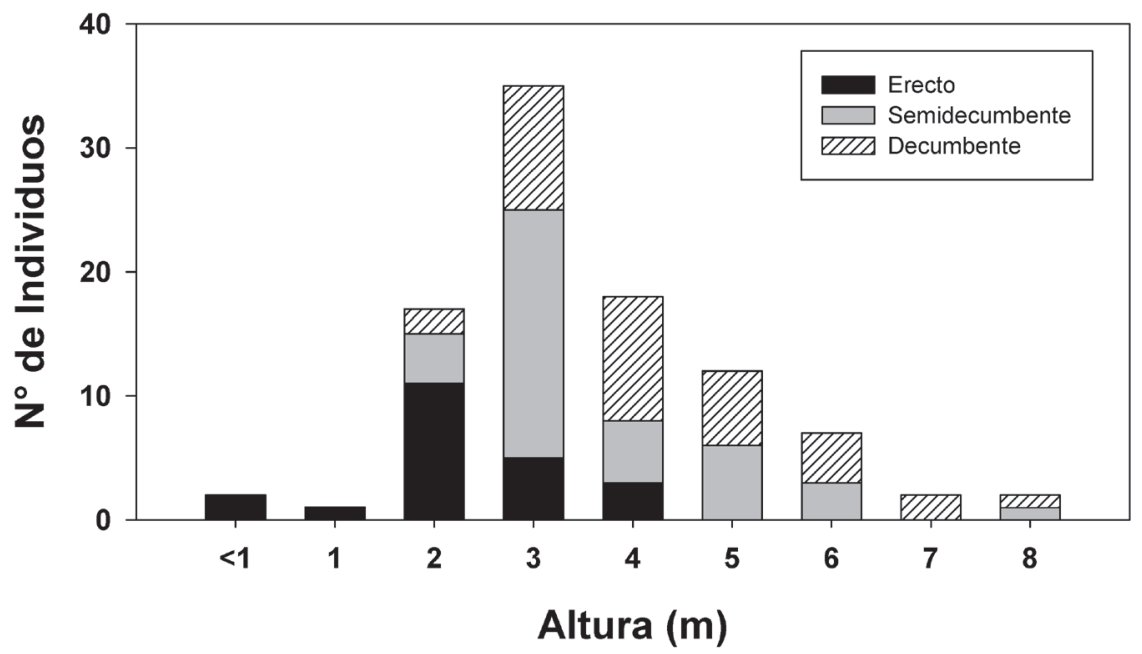

Fig. 2. Distribución de las alturas promedio del bosque Ramorinoa girolae y su forma según la altura. 


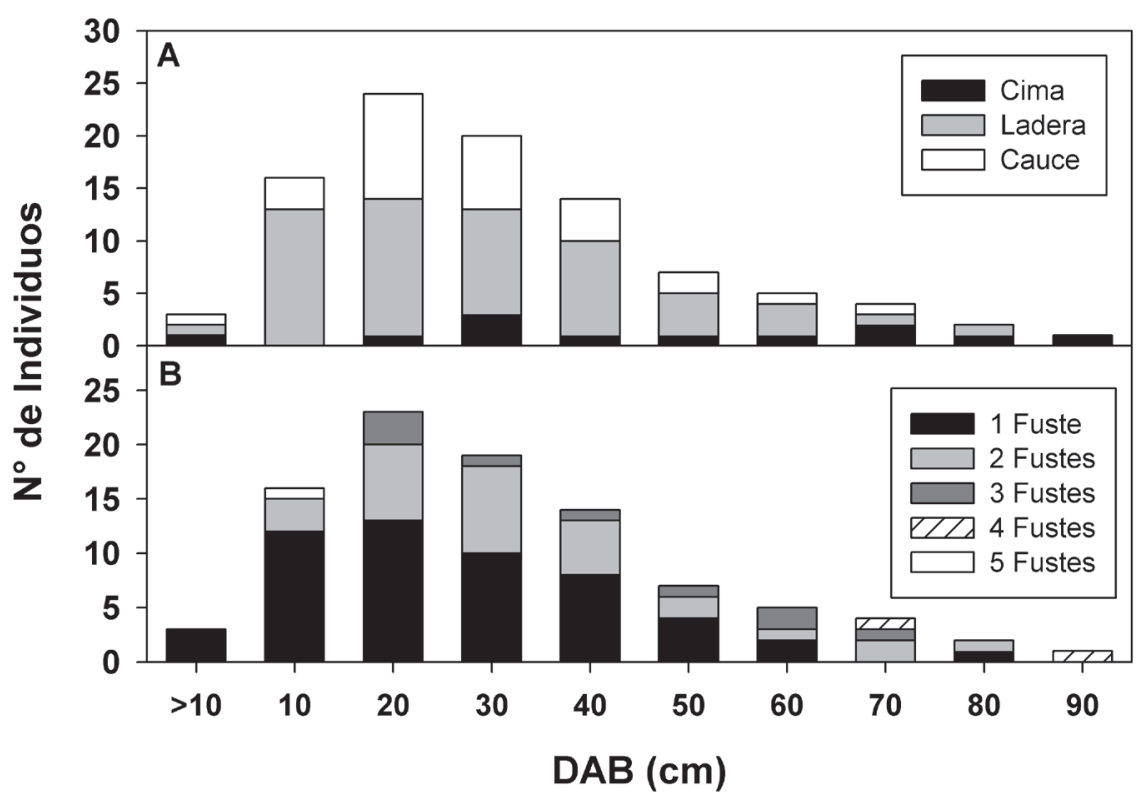

Fig. 3. Distribución diamétrica promedio del bosque de Ramorinoa girolae, relacionadas. A: Posición topográfica en la quebrada. B: Número de fustes de cada árbol.

$6,67 \pm 3,44 \mathrm{~m}$. Se registraron árboles con superficie de copa mayor a $200 \mathrm{~m}^{2}$, aunque el promedio fue de $54,02 \pm 52,83 \mathrm{~m}^{2}$. Cuando se relacionó el DAB con la superficie de copa, se observaron valores positivos y significativos $\left(R^{2}=0,32 ; F=45,08\right.$; $P=0,0001$; Fig. 5).

\section{Discusión y Conclusiones}

En el bosque estudiado de $R$. girolae en la Sierra de Pie de Palo, se puede observar que existió una regeneración casi continua, y se puede detectar que hay una regularidad en su distribución diamétrica. Sin embargo, la escasez de individuos menores a $10 \mathrm{~cm}$ de diámetro da indicios de que el bosque no se está regenerando en las últimas décadas. En el contexto de un cambio climático, las últimas estimaciones del Panel Intergubernamental sobre el Cambio Climático (IPCC), determinan que el $20-30 \%$ de las especies se encuentran en alto riesgo de extinción con un aumento de 2 a $3^{\circ} \mathrm{C}$ de temperatura (Brooke, 2008). En resultados obtenidos de ensayos con semillas de $R$. girolae se destaca el alto poder germinativo del tratamiento a $2{ }^{\circ} \mathrm{C}$ por $48 \mathrm{hs}$ (Meglioli et al., 2012), esta posible necesidad de las semillas de $R$. girolae de tener un periodo de baja temperaturas antes de la germinación y el aumento de la temperatura pueden ser que estén afectando la germinación y su posterior establecimiento. A su vez Meglioli et al. (2012) reportaron un bajo porcentaje de poder germinativo con el tratamiento mecánico, sin embargo Azagro Malo (2009) observó el poder germinativo de varias poblaciones de $R$. girolae, donde el tratamiento de escarificado mecánico obtuvo hasta un $92 \%$ de poder germinativo, aunque los porcentajes de germinación de las semillas de esta especie bajo un mismo tratamiento puede variar según la procedencia. En este sentido, si consideramos a $R$. girolae en categoría de conservación en peligro, sería pertinente dilucidar cómo afectaría el cambio climático a la germinación de esta especie, etapa que probablemente sea la más sensible.

Al igual que la estructura diamétrica, la distribución de la altura de los árboles de $R$. girolae, presenta un mayor número entre los árboles de 3 a 5 metros y reducida cantidad de individuos inferiores a un metro. Esta altura promedio es algo inferior a la observada en otro bosque de $R$. girolae en el Parque Provincial Ischigualasto, donde los 


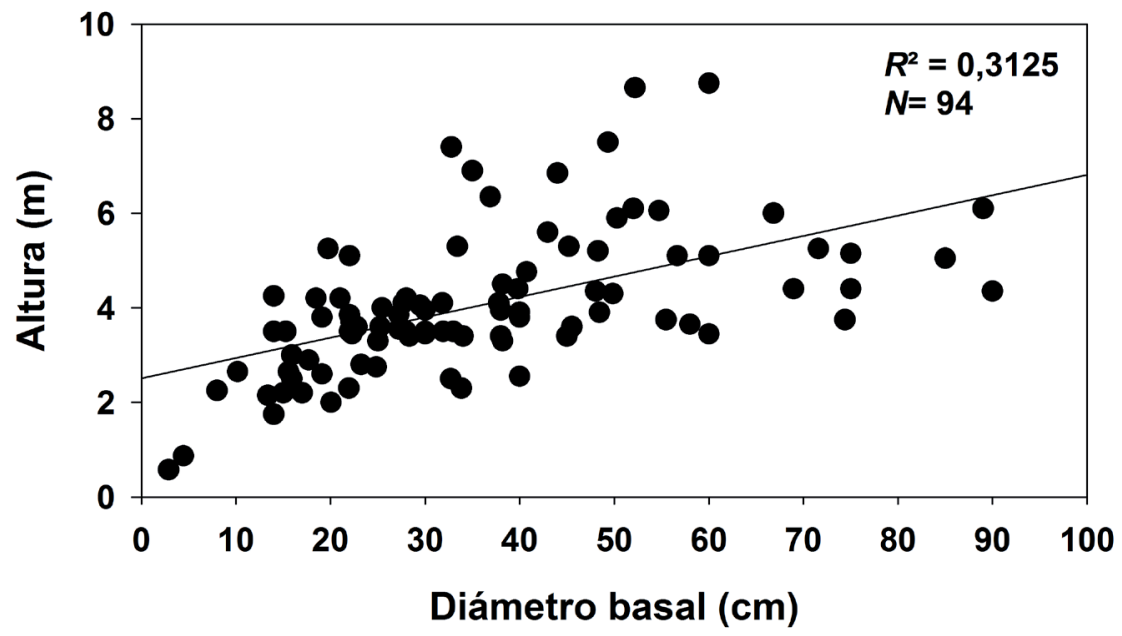

Fig. 4. Regresión lineal entre la altura de los árboles de $R$. girolae y el diámetro basal.

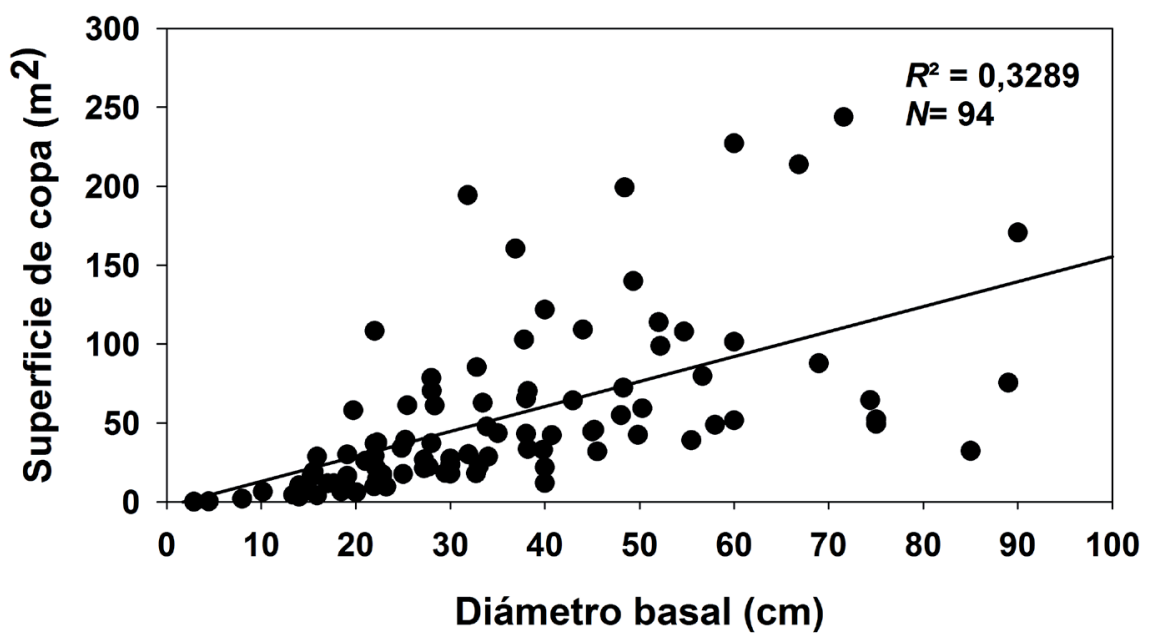

Fig. 5. Regresión lineal entre el diámetro basal de los árboles de R. girolae y la superficie de la copa.

árboles en general, poseen una altura de hasta 6 metros (Márquez et al., 2005), estás diferencias pueden estar dadas por las condiciones ambientales de cada sitio, como sugiere Villagra et al. (2005) para los bosques de Prosopis flexuosa a lo largo de un gradiente latitudinal. Sin embargo el promedio del diámetro mayor de copa de $8,33 \mathrm{~m}$ y menor de $6,67 \mathrm{~m}$ de $R$. girolae del presente trabajo fue semejante al observado por Márquez et al. (2005) para Ischigualasto, con un diámetro de copa entre 6 y $7 \mathrm{~m}$. Esta gran extensión de copa se ve reflejada en el promedio de la superficie de copa con 54,02 $\mathrm{m}^{2}$, con árboles que superan los $200 \mathrm{~m}^{2}$. Campos et al. (2013) observaron que esta gran extensión y densidad de la copa puede crear un microambiente que facilita el establecimiento y crecimiento de diferentes especies de plantas en los bosques de $R$. girolae en Ischigualasto, por lo que se la podría considerar una especie importante en el rol del ecosistema árido. 
Álvarez et al. (2006) observaron en bosques de Prosopis flexuosa D.C. estructuras abiertas o semicerradas con densidades que van de 181 árboles ha- ${ }^{-1}$ a 233 árboles ha $^{-1}$, respectivamente, mientras que en el presente trabajo observamos que el bosque de R. girloae tiene una densidad menor (117,5 árboles $\left.\mathrm{ha}^{-1}\right)$, por lo que se puede considerar como una estructura más abierta respecto a los bosques de $P$. flexuosa. Este trabajo es el primero que considera variables estructurales de $R$. girolae, por lo que posteriores estudios de este tipo podrían brindar información comparable entre sitios, acerca de la densidad y la estructura de las poblaciones.

Cuando se relacionó la altura y la forma, se observó que a partir de los $3 \mathrm{~m}$, esta especie adquiere una forma de semidecumbencia, posiblemente influenciada por el hábito de crecimiento. En este sentido, el incremento de las ramas y por ende, de la superficie de copa, conlleva a la decumbencia de las mismas, quizás debido a la elevada densidad de la madera (Kiesling, 1994) y su consecuente mayor peso que tira las ramas hacia abajo. A partir de las ramas que tocan el suelo pueden rebrotar nuevos individuos por reproducción agámica (obs. pers. de los autores). Esto merece una atención especial en el diseño metodológico de posteriores estudios ya que puede llevar a errores al considerar un solo individuo en lugar de dos o más, o viceversa. Sería de gran importancia hacer análisis de $\mathrm{ADN}$, para controlar como variable al parentesco genético existente entre los individuos de cada bosque y entre los bosques fragmentados, para mantener la independencia entre individuos en estudios poblacionales. Sumado a esto conocer la variabilidad genética intra e inter poblacional, con el fin de establecer estrategias de conservación. Además, los patrones genéticos histórico nos ofrecen la oportunidad de examinar cómo las especies han respondido a eventos climáticos anteriores en los plazos adecuados para la detección de los procesos ecológicos y evolutivos, como el flujo de genes, mutación, deriva genética y la selección natural (Wu et al., 1999).

En este trabajo se resalta un buen estado sanitario de la población de $R$. girolae, y del mayor número de árboles encontrados en ladera. Si bien como se mencionó anteriormente no se detectó muchos individuos jóvenes, parece ser que esta posición topográfica dentro de la quebrada encuentra una condición óptima para el establecimiento. Una hipótesis que se podría plantear es que en la ladera encuentra una mejor condición edáfica a diferencias del que se encuentra en la cima con suelos pedregosos. La diferencia que existe entre el número de individuos en laderas respecto al cauce puede estar dada por una mayor competencia con los arbustos de otras especies en el cauce, al existir mejores condiciones ambientales, para el establecimiento de posibles competidoras (Yeaton \& Cody, 1976; Yeaton, 1978; Conell, 1983; Schoener, 1983).

Estudios sobre las comunidades vegetales en el Parque Provincial Ischigualasto (Márquez et al. 2005), han observado que la recuperación natural de la cubierta vegetal es casi nula en áreas con $R$. girolae incendiadas. Este mismo tipo de disturbio se ha observado en bosques de $R$. girolae en áreas aledañas al sitio relevado donde no se observaron renovales. Comprender el efecto del fuego en comunidades vegetales que incluyan a $R$. girolae, puede ser de gran importancia en los procesos de sucesión que se producen en estos ambientes luego de este tipo de disturbio.

Es importante destacar que dentro de su restringida distribución natural, $R$. girolae se encuentra bajo diferentes áreas naturales protegidas, como es el caso en la Provincia de La Rioja, en el Parque Nacional Talampaya, en San Juan se puede encontrar bosques de $R$. girolae en el Parque Provincial Ischigualasto-Patrimonio de la Humanidad y en la Reserva de Usos Múltiples de Valle Fértil y en el Parque Nacional Sierra de las Quijadas en la Provincia de San Luis. Sería de suma importancia poder generar un plan de manejo que pueda contemplar toda la distribución de $R$. girolae así garantizar un buen desarrollo de estos bosques fragmentados per se.

En conclusión, en este trabajo se presentó un bosque de $R$. girolae en el extremo sur de la Sierra de Pie de Palo, donde se mostró características estructurales que permiten conocer mejor la situación actual de este bosque. A partir de estos resultados, se puede concluir que el bosque de $R$. girolae presenta una estructura diamétrica grande, lo que indica árboles viejos, sin embargo se observó un bajo número de árboles de diámetros menores a $10 \mathrm{~cm}$. El mayor número de árboles presentes en la ladera, nos permite pensar que en este tipo de topografía es donde se encuentran las mejores condiciones para el 
establecimiento, lo que indica que los factores ambientales pueden estar jugando un papel importante en la conformación de la estructura y regeneración dinámica en este bosque. Es importante destacar que el estado sanitario de la población es en general bueno. Consideramos de mucho valor biológico investigar con más detalle los patrones de regeneración de esta especie para conocer mejor su autoecología y poder plantear posteriores planes de manejo para su conservación in situ.

\section{Agradecimientos}

A los Téc. en Cs. Biol. Mauricio Pérez y José Carnino por la asistencia en campo y a los Lic. Yanina Ribas, Justo Marquez y al Dr. Alvarez, Juan por sus aportes a la corrección del manuscrito. Este trabajo está dedicado a la memoria de nuestro amigo y colega, Téc. en Cs. Biol. Mariano Gabriel Ariza (Sec. Medio Ambiente y Desarrollo sustentable, Gobierno de San Juan).

\section{Bibliografía}

Alvarez, J. A., P. VIllagra, M. A. CONY, E. M. CESCA, \& J. A. BONINSEGNA. 2006. Estructura y estado de conservación de los bosques de Prosopis flexuosa D.C. (Fabaceae, subfamilia: Mimosoideae) en el noreste de Mendoza (Argentina). Rev. Chil. Hist. Nat. 79: 75-87.

ARCHER, S. 1994. Regulation of ecosystem structure and function: climatic versus non-climatic factors. In: GRIFFITHS, J. E. (ed.), Handbook of Agricultural Meteorology. Oxford University Press, Oxford.

AZAGRO MALO, C. 2009. Germinación y obtención de plántulas de Ramorinoa girolae Speg. Especie leñosa endémica del centro oeste de la República Argentina. Tesina de Grado. Universidad Nacional de Córdoba.

BANNISTER, J. R., C. E. LE QUESNEA, \& A. LARA. 2008. Estructura y dinámica de bosques de Pilgerodendron uviferum afectados por incendios en la Cordillera de la Costa de la Isla Grande de Chiloé. Bosque 29: 33-43.

BEORCHIA NIGRIS, A. 2001. Los Chicales de Pie de Palo. In: San Juan Tierra de Huarpes. Tomo segundo. pp. 123-132. Editorial Papiro, San Juan.

BROOKE, C. 2008. Conservation and Adaptation to Climate Change. Conserv. Biol. 22: 1471-1476.
BROWN, P. M. \& R. Wu. 2005. Climate and disturbance forcing of episodic tree recruitment in a southwestern Ponderosa Pine Landscape. Ecology 86: 3030-3068.

CABRERA, A. L. 1994. Regiones fitogeográficas Argentinas. En: KUGLER, W. F. (ed.), Enciclopedia Argentina de Agricultura y Jardinería, $2^{\circ}$ ed., T II. F 1. Editorial Acme, Buenos Aires.

CAMPOS, V., P. BALMACEDA \& S. GIANNONI. 2013. Biological interaction in woodlands in the Monte desert. XXXI Reunión Anual de la Sociedad de Biología de Cuyo. 14 y 15 de noviembre, Mendoza. Argentina.

CANGIANO, M. A., L. A. ZANÍN \& H. N. LOSINNO. 1998. El Cariotipo de Ramorinoa girolae (Fabaceae). Kurtziana 26: 173-177.

CONELL, J. 1983. On the prevalence and relative importance of interspecific competition: evidence from field experiment. Am. Nat. 122: 661-96.

CULlEN, L. E., G. H. STEWART, R. P. DUNCAN \& J. G. PALMER. 2001. Disturbance and climate warming influences on New Zealand Nothofagus tree-line population dynamics. J. Ecol. 89:10611071 .

DEZZOTTI A. \& L. SANCHOLUZ. 1991. Los bosques de Austrocedrus chilensis en Argentina: ubicación, estructura y crecimiento. Bosque 12: 43-52.

DIRECCIÓN DE CONSERVACIÓN Y ÁREAS PROTEGIDAS, SECRETARIA DE AMBIENTE GOBIERNO DE SAN JUAN. 2009. Áreas Naturales Protegidas, Provincia de San Juan. Primera Edición.

DUNCAN, R. P. 1993. Flood disturbance and the coexistence of species in a lowland podocarp forest, Westland, New Zealand. J. Ecol. 81: 403-416.

DEMAIO, P., U. KARLIN \& M. MEDINA. 2002. Árboles nativos del centro de Argentina. Editorial L.O.L.A. (Literature of Latin America), Buenos Aires.

GIL, A. R. 2013. Estructura forestal y estado de conservación de los bosques de Prosopis chilensis y Prosopis flexuosa (algarrobales) de la Depresión del Río Bermejo, noreste de San Juan. Tesina de Grado. Universidad Nacional de Cuyo.

HUNZIKER, A. T. \& A. E. COCUCCI. 1961. Noticia sobre nuevas colecciones de Stenodrepanum bergii Harms. y Ramorinoa girolae Speg. (Leguminosae). Kurtziana 1: 307-308.

KIESLING, R. 1994. Flora de San Juan. Volumen I. Pteridófitas, Gimnospermas. Dicotiledóneas Dialipétalas (Salicáceas- Leguminosas). Vazquez Mazzini Editorial, Buenos Aires.

KIESLING, R. 2003. Flora de San Juan. Volumen II. Dicotiledóneas Dialipétalas (segunda parte: Oxalidáceas a Umbelíferas). Estudio Sigma, Buenos Aires. 
KIESLING, R., 2009. Flora de San Juan. Volumen IV. Monocotiledóneas. Editorial Fundación Universidad Nacional de San Juan. Zeta Editores, San Juan.

LABRAGA, J. C. \& R. Villalba. 2009. Climate in the Monte Desert: Past trends, present conditions, and future projections. J. Arid Environ. 73: 154-163.

LUNA, L. C., N. B. PIGNI, L. TORREAS-CLAVERIA, M. V. MONFERRAN, D. MAESTRI, D. A. WUNDERLIN, G. E. FERESIN, J. BASTIDA \& A. TAPIA. 2013. Ramorinoa girolae Speg (Fabaceae) seeds, an Argentinean traditional indigenous food: nutrient composition and antioxidant activity. $J$. Food Compos Anal. 31: 120-128.

MÁRQUEZ, J. 1999. Las áreas protegidas de la provincia de San Juan. Multequina 8: 1-10.

MÁRQUEZ, J., E. MARTINEZ CARRETERO, A. DALMASSO, G. PASTRÁN \& G. ORTIZ. 2005. Las áreas protegidas de la provincia de San Juan (Argentina) II. La vegetación del Parque Provicial Ischigualasto. Multequina 14: 1-27.

MARTÍNEZ DE ESCOBAR, S., Y. RIBAS FERNÁNDEZ, M. A. HADAD, P. A. NEIRA, L. SÁNCHEZ \& U. O. KARLIN. 2013. Estructura y estado de conservación del bosque mixto de Bulnesia retama y Prosopis spp. de la comunidad de Bermejo, Departamento Caucete, San Juan. 2013. IV Congreso Forestal Argentino y Latinoamericano. Misiones.

MEGLIOLI, C., J.A. SCAGLIA, M. HADAD, \& G. DÍAZ BISUTTI. 2012. Evaluación del poder germinativo de Ramorinoa girolae SPEG. (Fabaceae) bajo diferentes tratamientos pregerminativos. Análisis de Semillas 6: 62-65.

MORELLO, J. H. 1958. La Provincia Fitogeográfica del Monte. Opera Lilloana 2: 5-115.

NEWTON, A. C. 2007. Forest Ecology and Conservation. A Handbook of Techniques. Techniques in Ecology and Conservation Series, pp. 85-146. Oxford University Press, Oxford.

NORTH, M., J. CHEN, B. OAKLEY, B. SONG, M. RUDNICKI, A. GRAY \& J. INNES. 2004. Forest stand structure and pattern of old-growth western hemlock/Douglas-fir and Mixed- Conifer Forests. Forest Sci. 50: 299-311.

PASIECZNIK, N. M., P. FELKER, P. J. C. HARRIS, L. N. HARSH, G. CRUZ, J. C. TEWARI, K. CADORET \& L. J. MALDONADO. 2001. The Prosopis juliflora - Prosopis pallida complex: a Monograph. Henry Doubleday Research Association, Coventry, United Kingdom.

PERRY, D. A. 1994. Forest Ecosystems. The John Hopkins University Press, Baltimore-London.
ROMME W. H. \& D. H. KNIGHT. 1982. Landscape diversity: The concept applied to Yellowstone Park. Bioscience 32: 664-670.

SCHOENER, T. 1983. Field experiments on interspecific competition. Am. Nat. 122: 240-85.

SVENSSON, J. S. \& J. K. JEGLUM. 2001. Structure and dynamics of an undisturbed old-growth Norway Spruce forest on the rising Bothnian Coastline. Forest Ecol. Manag. 151: 67-79.

TAYLOR, A. H., Z. S. QIN \& L. JIE. 1996. Structure and dynamics of Subalpine forests in the Wang Lang Natural Reserve, Sichuan, China. Plant Ecol. 124: 25-38.

VEBLEN, T. T. 1992. Regeneration dynamics. In: GLENN-LEWIN, D., R. PEET, \& T. VEBLEN (eds.), Plant Succession: Theory and Prediction, pp. 152-187. Chapman \& Hall, London.

VILLAGRA, P.E., J.A. BONINSEGNA, J.A. ÁLVAREZ, M. CONY, E. CESCA, \& R, VILLALBA. 2005. Dendroecology of Prosopis flexuosa woodlands in the Monte desert : Implications for their management. Dendrocronologia 22: 209-213.

VILLEGAS, P. D., C. LE QUESNE, \& C. H. LUSK. 2003. Estructura y dinámica de una población de Gomortega keule (Mol.) Baillon en un rodal antiguo de bosque Valdiviano, Cordillera de Nahuelbuta, Chile. Gayana Bot. 60: 107-113.

WU, J., K. V. KRUTOVSKII \& S. H. STRAUSS. 1999. Nuclear DNA diversity, population differentiation, and phylogenetic relationships in the California closed-cone pines based on RAPD and allozyme markers. Genome 42: 893-908.

WANG, T., Y. LIANG, H. REN, D. YU, J. NI \& K. MA. 2004. Age structure of Picea schrenkiana forest along an altitudinal gradient in the central Tianshan Mountains, Northwestern China. Forest Ecol. Manag. 196: 267-274.

YEATON, R. 1978. A cyclical relationship between Larrea tridentata and Opuntia leptocaulis in the Northern Chihuahuan Desert. J. Ecol. 66: 651-6.

YEATON, R. \& M. CODY. 1976. Competition and spacing in plant communities: the Northern Mohave Desert. J. Ecol. 64: 689-96.

ZAPATA, R., P. R. QUIROGA \& N. R. GROSSO. 2010. Valoración química de la semillas de Ramorinoa girolae Speg. "chica” para la alimentación humana. Congreso Eco Productos Forestales No Madereros. Esquel.

Recibido el 13 de septiembre de 2013, aceptado el 20 de diciembre de 2013. 